Open J. Math. Anal., Vol. 2(2018), No. 1, pp. 66 - 73

Website: https://pisrt.org/psr-press/journals/oma/

ISSN: 2616-8111 (Online) 2616-8103 (Print)

http://dx.doi.org/10.30538/psrp-oma2018.0012

\title{
ZAGREB POLYNOMIALS AND REDEFINED ZAGREB INDICES FOR THE LINE GRAPH OF CARBON NANOCONES
}

\author{
SABA NOREEN ${ }^{1}$, ATIF MAHMOOD
}

\begin{abstract}
A line graph has many useful applications in physical chemistry. Topological indices are numerical parameters associated to a structure and, in combination, determine properties of the concerned material. In this paper, we compute the closed form of Zagreb polynomilas of all generalized class of carbon nanocones and compute important degree-based topological indices.
\end{abstract}

AMS Mathematics Subject Classification: 05C05, 05C07, 05C35.

Key words and phrases: Zagreb polynomial; degree-based index; Carbon nanocone

\section{Introduction}

Chemical graph theory is a subject which connects Mathematics, chemistry and graph theory. A topological index is a numeric number associated with molecular graph and this number correlate certain physico-chemical properties of chemical compounds. The topological indices such as the Wiener index, first and second Zagreb index, modified Zagreb index, Randic index and symmetric division index, Harmonic index, Invers sum index, Augmented Zagreb index, etc. are useful in prediction of bioactivity of the chemical compounds [1, 2, 3, 4, 5. These indices capture the overall structure of compound and predict chemical properties such as strain energy, heat of formation, and boiling points etc. Carbon nanocones have been observed since 1968 or even earlier [6], on the surface of naturally occurring graphite. The importance of carbon nanostructures is due to their potential use in many applications including gas sensors, energy storage, nanoelectronic devices, biosensors and chemical probes [7. Carbon allotropes

Received 09-04-2018. Revised 30-05-2018.

1 Corresponding Author

(C) 2018 Saba Noreen, Atif Mahmood. This is an open access article distributed under the Creative Commons Attribution License, which permits unrestricted use, distribution, and reproduction in any medium, provided the original work is properly cited. 
such as carbon nanocones and carbon nanotubes have been proposed as possible molecular gas storage devices [8, 9. More recently, carbon nanocones have gained increased scientific interest due to their unique properties and promising uses in many novel applications such as energy and hydrogen-storage [10. Figure 1 and Figure 2 are carbon nenocones.

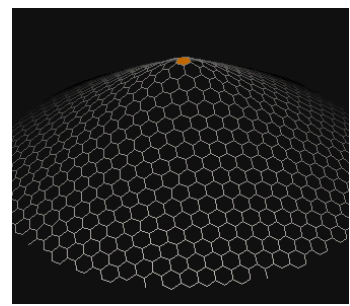

Figure 1. Carbon Nenocone $C N C_{k}[5]$

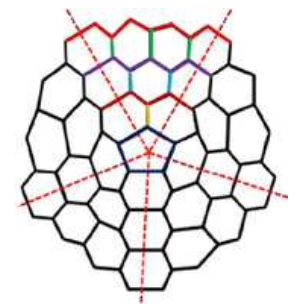

Figure 2. The Molecular graph of $C N C_{k}[5]$

The molecular graph of $C N C_{k}[n]$ nanocones have conical structures with a cycle of length $k$ at its core and $n$ layers of hexagons placed at the conical surface around its center as shown in following Figure 3.

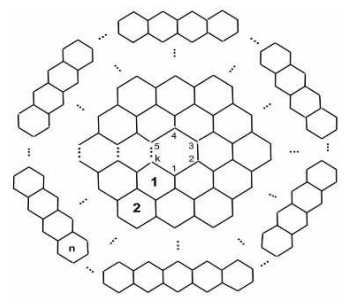

Figure 3. Carbon nenocone $C N C_{k}[n]$

The line graph $L(G)$ of a graph $G$ is the graph each of whose vertex represents an edge of $G$ [11, 12, 13, 14] and two of its vertices are adjacent if their corresponding edges are adjacent in $G$. 


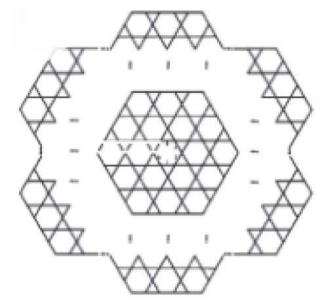

Figure 4. The line graph ofCarbon nenocone $C N C_{k}[n]$

In the present report, we gave closed form of Zagreb-polynomials of Carbon nenocones. We also compute some degree-based topological indices. In [15, authors computed Hosoya polynomial and related distance based indices for $\mathrm{CNC}_{7}[n]$. In [16] authors computed the Vertex PI, Szeged and Omega Polynomials of Carbon Nanocones $C N_{4}[n]$. Similarly many partial results about topological indices have been obtained about some particular classes of Nanocones. We however present most general results about complete families of nanocones. Our results present nice generalizations of many existing partial results.

Let $G$ be a connected graph. The vertex and edge sets are denoted by $V(G)$ and $E(G)$, respectively. For every vertex $v \in V(G)$, degree of $v$ is number of vertices attached with it. The first and the second Zagreb indices (cf. [17) are defined as

$$
M_{1}(G)=\sum_{u v \in E(G)}\left(d_{u}+d_{v}\right)
$$

and

$$
M_{2}(G)=\sum_{u v \in E(G)}\left(d_{u} \cdot d_{v}\right) .
$$

Considering the Zagreb indices, Fath-Tabar ([18]) defined first and the second Zagreb polynomials

$$
M_{1}(G, x)=\sum_{u v \in E(G)} x^{d_{u}+d_{v}}
$$

and

$$
M_{2}(G, x)=\sum_{u v \in E(G)} x^{d_{u} \cdot d_{v}} .
$$

The properties of first and second Zagreb polynomials for some chemical structures have been studied in the literature [19]. After that, in [20, the authors defined the third Zagreb index

$$
M_{3}(G)=\sum_{u v \in E(G)}\left(d_{u}-d_{v}\right)
$$

and Zagreb polynomials

$$
M_{3}(G, x)=\sum_{u v \in E(G)} x^{d_{u}-d_{v}} .
$$

In the year 2016, 21] following Zagreb type polynomials were defined 
- $M_{4}(G, x)=\sum_{u v \in E(G)} x^{d_{u}\left(d_{u}+d_{v}\right)}$

- $M_{5}(G, x)=\sum_{u v \in E(G)} x^{d_{v}\left(d_{u}+d_{v}\right)}$

- $M_{a, b}(G, x)=\sum_{u v \in E(G)} x^{a d_{u}+b d_{v}}$,

- $M_{a, b}^{\prime}(G, x)=\sum_{u v \in E(G)} x^{\left(a+d_{u}\right)+\left(b+d_{v}\right)}$.

Ranjini et al. 22 redefines the Zagreb index, i. e, the redefined first, second and third Zagreb indices of graph $G$. These indicators appear as

- $\operatorname{Re} Z G_{1}(G)=\sum_{u v \in E(G)} \frac{d_{u}+d_{v}}{d_{u} d_{v}}$

- $\operatorname{Re} Z G_{2}(G)=\sum_{u v \in E(G)} \frac{d_{u} d_{v}}{d_{u}+d_{v}}$

- $\operatorname{Re} Z G_{3}(G)=\sum_{u v \in E(G)}\left(d_{u} \cdot d_{v}\right)\left(d_{u}+d_{v}\right)$.

\section{Main Results}

In this section, we will present our computational results

Theorem 2.1. Let $L\left(C N C_{k}[n]\right)$ be the line graph of Carbon Nanocones $C N C_{k}[n]$ . Then

(1) $M_{1}\left(L\left(C N C_{k}[n]\right), x\right)=2 k x^{5}+k(2 n-1) x^{6}+2 k n x^{7}+3 k n^{2} x^{8}$

(2) $M_{2}\left(L\left(C N C_{k}[n]\right), x\right)=2 k x^{6}+k(2 n-1) x^{9}+2 k n x^{12}+3 k n^{2} x^{16}$

(3) $M_{3}\left(L\left(C N C_{k}[n]\right), x\right)=k\left[3 n^{2}+2 n-1\right]+2 k(n+1) x$

(4) $M_{4}\left(L\left(C N C_{k}[n]\right), x\right)=2 k x^{10}+k(2 n-1) x^{18}+2 k n x^{21}+3 k n^{2} x^{32}$

(5) $M_{5}\left(L\left(C N C_{k}[n]\right), x\right)=2 k x^{15}+k(2 n-1) x^{18}+2 k n x^{21}+3 k n^{2} x^{32}$

(6) $M_{a, b}\left(L\left(C N C_{k}[n]\right), x\right)=2 k x^{(2 a+3 b)}+k(2 n-1) x^{3(a+b)}$ $+2 k n x^{(3 a+4 b)}+3 k n^{2} x^{4(a+b)}$

(7) $M_{a, b}^{\prime}\left(L\left(C N C_{k}[n]\right), x\right)=2 k x^{(a+2)(b+3)}+k(2 n-1) x^{(a+2)(b+3)}$

$+2 k n x^{(a+2)(b+3)}+3 k n^{2} x^{(a+2)(b+3)}$

Proof. Let $L\left(C N C_{k}[n]\right)$ be the line graph of Carbon Nanocones $C N C_{k}[n]$. From the graph of $C N C_{k}[n]$ (figure 4) we can see that the total number of vertices are $8 k+2 k n$ and total number of edges are $k(n+1)(3 n+1)$. The edge set of $L\left(C N C_{k}[n]\right)$ has following four partitions

$E_{1}=E_{\{2,3\}}=\left\{e=u v \in L\left(C N C_{k}[n]\right): d_{u}=2, d_{v}=3\right\}$,

$E_{2}=E_{\{3,3\}}=\left\{e=u v \in L\left(C N C_{k}[n]\right): d_{u}=3, d_{v}=3\right\}$,

$E_{3}=E_{\{3,4\}}=\left\{e=u v \in L\left(C N C_{k}[n]\right): d_{u}=3, d_{v}=4\right\}$,

and

$E_{4}=E_{\{4,4\}}=\left\{e=u v \in L\left(C N C_{k}[n]\right): d_{u}=4, d_{v}=4\right\}$.

Now,

$\left|E_{1}\left(L\left(C N C_{k}[n]\right)\right)\right|=2 k$,

$\left|E_{2}\left(L\left(C N C_{k}[n]\right)\right)\right|=k(2 n-1)$,

$\left|E_{3}\left(L\left(C N C_{k}[n]\right)\right)\right|=2 k n$, 
and

$$
\left|E_{4}\left(L\left(C N C_{k}[n]\right)\right)\right|=3 k n^{2} .
$$

$$
\begin{aligned}
M_{1}\left(L\left(C N C_{k}[n]\right), x\right)= & \sum_{u v \in E\left(L\left(C N C_{k}[n]\right)\right)} x^{d_{u}+d_{v}} \\
= & \left|E_{1}\left(L\left(C N C_{k}[n]\right)\right)\right| x^{5}+\left|E_{2}\left(L\left(C N C_{k}[n]\right)\right)\right| x^{6} \\
& +\left|E_{3}\left(L\left(C N C_{k}[n]\right)\right)\right| x^{7}+\left|E_{4}\left(L\left(C N C_{k}[n]\right)\right)\right| x^{8} \\
= & 2 k x^{5}+k(2 n-1) x^{6}+2 k n x^{7}+3 k n^{2} x^{8} .
\end{aligned}
$$

(2)

$$
\begin{aligned}
M_{2}\left(L\left(C N C_{k}[n]\right), x\right)= & \sum_{u v \in E\left(L\left(C N C_{k}[n]\right)\right)} x^{d_{u} \times d_{v}} \\
= & \left|E_{1}\left(L\left(C N C_{k}[n]\right)\right)\right| x^{6}+\left|E_{2}\left(L\left(C N C_{k}[n]\right)\right)\right| x^{9} \\
& +\left|E_{3}\left(L\left(C N C_{k}[n]\right)\right)\right| x^{12}+\left|E_{4}\left(L\left(C N C_{k}[n]\right)\right)\right| x^{16} \\
= & 2 k x^{6}+k(2 n-1) x^{9}+2 k n x^{12}+3 k n^{2} x^{16} .
\end{aligned}
$$

(3)

$$
\begin{aligned}
M_{3}\left(L\left(C N C_{k}[n]\right), x\right)= & \sum_{u v \in E\left(L\left(C N C_{k}[n]\right)\right)} x^{\left|d_{u}-d_{v}\right|} \\
= & \left|E_{1}\left(L\left(C N C_{k}[n]\right)\right)\right| x+\left|E_{2}\left(L\left(C N C_{k}[n]\right)\right)\right| \\
& +\left|E_{3}\left(L\left(C N C_{k}[n]\right)\right)\right| x+\left|E_{4}\left(L\left(C N C_{k}[n]\right)\right)\right| \\
= & k\left[3 n^{2}+2 n-1\right]+2 k(n+1) x .
\end{aligned}
$$

(4)

$$
\begin{aligned}
M_{4}\left(L\left(C N C_{k}[n]\right), x\right)= & \sum_{u v \in E\left(L\left(C N C_{k}[n]\right)\right)} x^{d_{u}\left(d_{u}+d_{v}\right)} \\
= & \left|E_{1}\left(L\left(C N C_{k}[n]\right)\right)\right| x^{10}+\left|E_{2}\left(L\left(C N C_{k}[n]\right)\right)\right| x^{18} \\
& +\left|E_{3}\left(L\left(C N C_{k}[n]\right)\right)\right| x^{21}+\left|E_{4}\left(L\left(C N C_{k}[n]\right)\right)\right| x^{32} \\
= & 2 k x^{10}+k(2 n-1) x^{18}+2 k n x^{21}+3 k n^{2} x^{32} .
\end{aligned}
$$

(5)

$$
\begin{aligned}
M_{5}\left(L\left(C N C_{k}[n]\right), x\right)= & \sum_{u v \in E\left(L\left(C N C_{k}[n]\right)\right)} x^{d_{v}\left(d_{u}+d_{v}\right)} \\
= & \left|E_{1}\left(L\left(C N C_{k}[n]\right)\right)\right| x^{15}+\left|E_{2}\left(L\left(C N C_{k}[n]\right)\right)\right| x^{18} \\
& +\left|E_{3}\left(L\left(C N C_{k}[n]\right)\right)\right| x^{28}+\left|E_{4}\left(L\left(C N C_{k}[n]\right)\right)\right| x^{32} \\
= & 2 k x^{15}+k(2 n-1) x^{18}+2 k n x^{28}+3 k n^{2} x^{32} .
\end{aligned}
$$


(6)

$$
\begin{aligned}
M_{a, b}\left(L\left(C N C_{k}[n]\right), x\right)= & \sum_{u v \in E\left(L\left(C N C_{k}[n]\right)\right)} x^{a d_{u}+b d_{v}} \\
= & \left|E_{1}\left(L\left(C N C_{k}[n]\right)\right)\right| x^{2 a+3 b}+\left|E_{2}\left(L\left(C N C_{k}[n]\right)\right)\right| x^{3 a+3 b} \\
& +\left|E_{3}\left(L\left(C N C_{k}[n]\right)\right)\right| x^{3 a+3 b}+\left|E_{4}\left(L\left(C N C_{k}[n]\right)\right)\right| x^{4 a+4 b} \\
= & 2 k x^{(2 a+3 b)}+k(2 n-1) x^{3(a+b)} \\
& +2 k n x^{(3 a+4 b)}+3 k n^{2} x^{4(a+b)} .
\end{aligned}
$$

(7)

$$
\begin{aligned}
& M_{a, b}^{\prime}\left(L\left(C N C_{k}[n]\right), x\right) \\
= & \sum_{u v \in E\left(9 L\left(C N C_{k}[n]\right)\right)} x^{\left(a+d_{u}\right)+\left(b+d_{v}\right)} \\
= & \left|E_{1}\left(L\left(C N C_{k}[n]\right)\right)\right| x^{(a+2)+(b+3)}+\left|E_{2}\left(L\left(C N C_{k}[n]\right)\right)\right| x^{(a+3)+(3+b)} \\
& +\left|E_{3}\left(L\left(C N C_{k}[n]\right)\right)\right| x^{(a+3)+(4+b)}+\left|E_{4}\left(L\left(C N C_{k}[n]\right)\right)\right| x^{(4+a)+(4+b)} \\
= & 2 k x^{(a+2)(b+3)}+k(2 n-1) x^{(a+2)(b+3)}+2 k n x^{(a+2)(b+3)}+3 k n^{2} x^{(a+2)(b+3)} .
\end{aligned}
$$

Theorem 2.2. Let $L\left(C N C_{k}[n]\right)$ be the line graph of Carbon Nanocones $C N C_{k}[n]$. Then,

(1) $\operatorname{ReZ} G_{1}\left(L\left(C N C_{k}[n]\right)\right)=\frac{3}{2} k n^{2}+\frac{5}{2} k n+k$,

(2) $R e Z G_{2}\left(L\left(C N C_{k}[n]\right)\right)=6 k k n^{2}+\frac{45}{7} k n+\frac{9}{10} k$,

(3) $\operatorname{ReZ}_{3}\left(L\left(C N C_{k}[n]\right)\right)=6 k\left(64 n^{2}+46 n+1\right)$.

Proof. (1)

$$
\begin{aligned}
R e Z G_{1}\left(L\left(C N C_{k}[n]\right)\right)= & \sum_{u v \in E\left(L\left(C N C_{k}[n]\right)\right)} \frac{d_{u}+d_{v}}{d_{u} \cdot d_{v}} \\
= & \left|E_{1}\left(L\left(C N C_{k}[n]\right)\right)\right| \frac{5}{6}+\left|E_{2}\left(L\left(C N C_{k}[n]\right)\right)\right| \frac{6}{9} \\
& +\left|E_{3}\left(L\left(C N C_{k}[n]\right)\right)\right| \frac{7}{12}+\left|E_{4}\left(L\left(C N C_{k}[n]\right)\right)\right| \frac{8}{16} \\
= & (2 k) \frac{5}{6}+k(2 n-1) \frac{6}{9}+(2 k n) \frac{7}{12}+\left(3 k n^{2}\right) \frac{1}{2} \\
= & \frac{5}{3} k+\frac{4}{3} k n-\frac{2}{3} k+\frac{7}{6} k n+\frac{1}{2} k n^{2} \\
= & \frac{3}{2} k n^{2}+\left(\frac{24+21}{18}\right) k n+\left(\frac{5-2}{3}\right) k \\
= & \frac{3}{2} k n^{2}+\frac{5}{2} k n+k .
\end{aligned}
$$


$(2)$

$$
\begin{aligned}
R e Z G_{2}\left(L\left(C N C_{k}[n]\right), x\right)= & \sum_{u v \in E\left(L\left(C N C_{k}[n]\right)\right)} \frac{d_{u} \cdot d_{v}}{d_{u}+d_{v}} \\
= & \left|E_{1}\left(L\left(C N C_{k}[n]\right)\right)\right| \frac{6}{5}+\left|E_{2}\left(L\left(C N C_{k}[n]\right)\right)\right| \frac{9}{6} \\
& +\left|E_{3}\left(L\left(C N C_{k}[n]\right)\right)\right| \frac{12}{7}+\left|E_{4}\left(L\left(C N C_{k}[n]\right)\right)\right| \frac{16}{8} \\
= & (2 k) \frac{6}{5}+k(2 n-1) \frac{9}{6}+(2 k n) \frac{12}{6}+2\left(3 k n^{2}\right) \\
= & \frac{12}{5} k+3 k n-\frac{3}{2} k+\frac{24}{7} k n+6 k n^{2} \\
= & 6 k n^{2}+\left(\frac{24+21}{7}\right) k n+\left(\frac{24-15}{10}\right) k \\
= & 6 k k n^{2}+\frac{45}{7} k n+\frac{9}{10} k .
\end{aligned}
$$

(3)

$$
\begin{aligned}
\operatorname{ReZG}_{3}\left(L\left(C N C_{k}[n]\right), x\right)= & \sum_{u v \in E\left(L\left(C N C_{k}[n]\right)\right)}\left(d_{u} . d_{v}\right)\left(d_{u}+d_{v}\right) \\
= & \left|E_{1}\left(L\left(C N C_{k}[n]\right)\right)\right| 30+\left|E_{2}\left(L\left(C N C_{k}[n]\right)\right)\right| 54 \\
& +\left|E_{3}\left(L\left(C N C_{k}[n]\right)\right)\right| 84+\left|E_{4}\left(L\left(C N C_{k}[n]\right)\right)\right| 128 \\
= & (2 k) \frac{5}{6}+k(2 n-1) \frac{6}{9}+(2 k n) \frac{7}{12}+\left(3 k n^{2}\right) \frac{1}{2} \\
= & 30(2 k)+54 k(2 n-1)+84(2 k n)+128\left(3 k n^{2}\right) \\
= & 60 k+108 k n-54 k+168 k n+384 k n^{2} \\
= & 6 k\left(64 n^{2}+46 n+1\right) .
\end{aligned}
$$

\section{Competing Interests}

The author(s) do not have any competing interests in the manuscript.

\section{REFERENCES}

1. Basak, S. C., Mills, D., Mumtaz, M. M., \& Balasubramanian, K. (2003). Use of topological indices in predicting aryl hydrocarbon receptor binding potency of dibenzofurans: A hierarchical QSAR approach.

2. Garca, I., Fall, Y., \& Gómez, G. (2010). Using topological indices to predict anti-Alzheimer and anti-parasitic GSK-3 inhibitors by multi-target QSAR in silico screening. Molecules, 15(8), 5408-5422.

3. Verma, S., Le Bras, J., Jain, S. L., \& Muzart, J. (2013). Thiol-yne click on nano-starch: An expedient approach for grafting of oxo-vanadium Schiff base catalyst and its use in the oxidation of alcohols. Applied Catalysis A: General, 468, 334-340. 
4. Verma, S., Le Bras, J., Jain, S. L., \& Muzart, J. (2013). Nanocrystalline starch grafted palladium (II) complex for the MizorokiHeck reaction. Dalton Transactions, 42(40), 1445414459.

5. Verma, S., Tripathi, D., Gupta, P., Singh, R., Bahuguna, G. M., Chauhan, R. K., Saran, S., \& Jain, S. L. (2013). Highly dispersed palladium nanoparticles grafted onto nanocrystalline starch for the oxidation of alcohols using molecular oxygen as an oxidant. Dalton Transactions, 42(32), 11522-11527.

6. Gillot, J., Bollmann, W., \& Lux, B. (1968). Cristaux de graphite en forme de cigare et a structure conique. Carbon, 6(3), 381-387.

7. Iijima, S. (1991). Helical microtubules of graphitic carbon. nature, 354(6348), 56.

8. Adisa, O. O., Cox, B. J., \& Hill, J. M. (2011). Modelling the surface adsorption of methane on carbon nanostructures. Carbon, 49(10), 3212-3218.

9. Zhao, J., Buldum, A., Han, J., \& Lu, J. P. (2002). Gas molecule adsorption in carbon nanotubes and nanotube bundles. Nanotechnology, 13(2), 195.

10. Li, X., \& Gutman, I. Mathematical Aspects of Randic-Type Molecular Structure Descriptors, Mathematical Chemistry Monographs No. 1, Kragujevac, 2006, pp. V I, 330.

11. Wu, B. (2010). Wiener index of line graphs. MATCH Commun. Math. Comput. Chem, 64(3), 699-706.

12. Buckley, F., \& Superville, L. (1981). Mean distance in line graphs. Congr. Numer, 32(1), 153162 .

13. Huo, Y., Liu, J. B., Zahid, Z., Zafar, S., Farahani, M. R., \& Nadeem, M. F. (2016). On Certain Topological Indices of the Line Graph of $C N C k[n]$ Nanocones. Journal of Computational and Theoretical Nanoscience, 13(7), 4318-4322.

14. Iranmanesh, A., Gutman, I., Khormali, O., \& Mahmiani, A. (2009). The edge versions of the Wiener index. Match. MATCH Commun. Math. Comput. Chem, 61(3), 663-672.

15. Xu, S. J., \& Zhang, Q. X. (2013). The Hosoya Polynomial of One-Heptagonal Nanocone. Current Nanoscience, 9(3), 411-414.

16. Ghorbani, M., \& Jalali, M. (2009). The Vertex PI, Szeged and Omega Polynomials of Carbon Nanocones $\mathrm{CNC}_{4}(n)$. MATCH Commun. Math. Comput. Chem, 62(2), 353-362.

17. Gutman, I., \& Das, K. C. (2004). The first Zagreb index 30 years after. MATCH Commun. Math. Comput. Chem, 50, 83-92.

18. Fath-Tabar, G. H. (2011). Old and new Zagreb indices of graphs. MATCH Commun. Math. Comput. Chem, 65(1), 79-84.

19. Ranjini, P. S., Lokesha, V., Bindusree, A. R., \& Raju, M. P. (2012). New Bounds on Zagreb indices and the Zagreb Co-indices. Boletim da Sociedade Paranaense de Matemtica, 31(1), 51-55.

20. Fath-Tabar, G. (2009). Zagreb polynomial and Pi indices of some Nano Structures. Digest Journal of Nanomaterials \& Biostructures (DJNB), 4(1),189-191.

21. Bindusree, A. R., Cangul, I. N., Lokesha, V., \& Cevik, A. S. (2016). Zagreb polynomials of three graph operators. Filomat, 30(7), 1979-1986.

22. Ranjini, P. S., Lokesha, V., \& Usha, A. (2013). Relation between phenylene and hexagonal squeeze using harmonic index. International Journal of Graph Theory, 1(4), 116-121.

\section{Saba Noreen}

Department of Mathematics and Statistics, The University of Lahore, Lahore Pakistan.

e-mail: sabaalvi77@yahoo.com

\section{Atif Mahmood}

Department of Mathematics and Statistics, The University of Lahore, (Pakpattan Campus) Lahore Pakistan.

e-mail: atifdps@gmail.com 